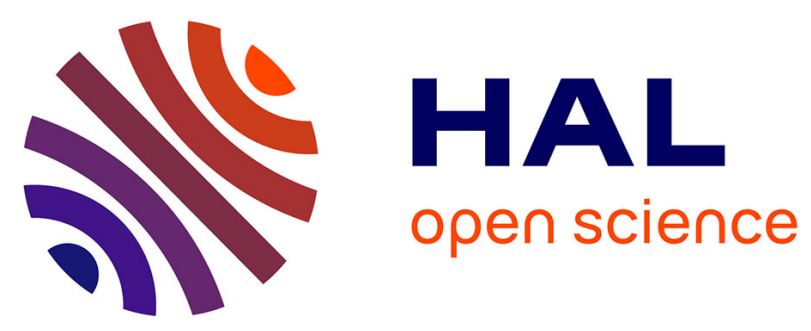

\title{
Simultaneous underwater acoustic localization and communication: an experimental study
}

Raphaël Garin, Charles Vanwynsberghe, Philippe Forjonel, Beatrice Tomasi, Pierre-Jean Bouvet

\section{- To cite this version:}

Raphaël Garin, Charles Vanwynsberghe, Philippe Forjonel, Beatrice Tomasi, Pierre-Jean Bouvet. Simultaneous underwater acoustic localization and communication: an experimental study. OCEANS 2021, Sep 2021, Porto, Portugal. hal-03456667

\section{HAL Id: hal-03456667 https://hal.science/hal-03456667}

Submitted on 30 Nov 2021

HAL is a multi-disciplinary open access archive for the deposit and dissemination of scientific research documents, whether they are published or not. The documents may come from teaching and research institutions in France or abroad, or from public or private research centers.
L'archive ouverte pluridisciplinaire HAL, est destinée au dépôt et à la diffusion de documents scientifiques de niveau recherche, publiés ou non, émanant des établissements d'enseignement et de recherche français ou étrangers, des laboratoires publics ou privés. 


\section{Simultaneous underwater acoustic localization and communication: an experimental study}

\author{
Raphaël Garin \\ L@bISEN \\ ISEN Yncréa Ouest \\ Brest, France \\ raphael.garin@isen-ouest.yncrea.fr \\ Beatrice Tomasi \\ L@bISEN \\ ISEN Yncréa Ouest \\ Brest, France \\ beatrice.tomasi@isen-ouest.yncrea.fr
}

\author{
Charles Vanwynsberghe \\ L@bISEN \\ ISEN Yncréa Ouest \\ Brest, France \\ charles.vanwynsberghe@isen-ouest.yncrea.fr
}

\author{
Philippe Forjonel \\ L@bISEN \\ ISEN Yncréa Ouest \\ Brest, France
}

philippe.forjonel@isen-ouest.yncrea.fr

\begin{abstract}
Currently in the field of autonomous underwater vehicles localization, it is difficult to do without a Doppler Velocity Log (DVL). Combined with distance measurements or not, this fairly expensive sensor is a must-have. In this paper we introduce our method by combining underwater acoustic communication and localization through Doppler shift estimation needed in the vehicle transmission modem for communication. This cost-effective technique provides an information on transmitter/receiver relative speed. We consider a fixed anchor with known position transmitting regularly to a receiver in a water pool of $3 \mathrm{~m}^{2}$. Using an Extended Kalman filter, we compare standalone range measurements estimation with speed-only measurements and speed with range combined in the estimation filter. Experimental results demonstrate a consequent gain using speed computation associated with range measurement.

Index Terms-Underwater navigation, EKF, underwater acoustic communications, Doppler shift estimation, Autonomous underwater vehicle.
\end{abstract}

\section{INTRODUCTION AND MOTIVATIONS}

Autonomous underwater vehicles have been developed since the early 1970s and are nowadays intensively exploited to perform tasks previously made by manned vehicles. They are employed in scientific cruises, e.g., to carry on geological surveys or underwater wildlife mapping, but also in numerous military and commercial applications. The Autonomous Underwater Vehicle (AUV) navigation system relies on the precision and the accuracy of its own localization.

In many use cases, the AUV needs to both navigate and communicate with an operating platform, e.g., a beacon or another AUV. Underwater communication ranges are from 1 centimeter to few kilometers in shallow water, neither optical or radio-frequency systems are suitable since radio waves attenuate rapidly in salt water $(1800 \mathrm{~dB} / \mathrm{m})$. Therefore, radio systems such as GPS or GSM, cannot be used. Waves at visible light frequencies can reach up to $100 \mathrm{~m}$ range but typical applications require a distance between the AUV and the operating platform of several hundreds meters. Underwater Acoustic (UWA) channel is then the best option for simultaneous communication and localization thanks to its best performance compared to others.

Although UWA channels are characterized by high latency (due to sound speed of approximately $1500 \mathrm{~m} / \mathrm{s}$ ), low data rate (about $5 \mathrm{kbps}$ ) and possible long multipath that exhibit significant technical challenges [1].

\section{A. Localization methods}

In this paper, we focuse on a new localization method. The scientific community has researched on novel AUV localization methods in the past ten years leading to several innovative technologies in this domain, e.g., in [2] and [3].

Industries have also developed localization methods for AUVs. In particular, two technologies have proven their effectiveness :

- Transponder-based systems : a single fixed beacon sends a periodic acoustic ping to measure the distance between the beacon and the AUV (range measurement), Long Baseline (LBL), Short Baseline (SBL) and Ultra Short Baseline (USBL).

These systems provide accurate positioning but they require calibration and precisely localized infrastructures, which reduces flexibility and increases the deployment time.

- Inertial or dead reckoning systems: these include a DVL to compute velocity, and an Inertial Mesurement Unit (IMU) to compute acceleration, angular velocities, orientation, heading.

Most of the time, AUVs use these systems, since they are standalone systems and can guarantee satisfactory localization performance. However, they are expensive and the IMU sensor is affected by a drift across time which implies a bias, thus reducing the localization accuracy.

These two technologies are complementary and when used jointly they can make localization methods more accurate and 
precise. An AUV could also integrate sensors as compass, pressure sensor, water speed sensor or magnetometer that could feed complementary information into the localization estimator to improve its performance. However, this is out the scope of this paper. Transponders and GPS surface initialization corrects the drift of inertial systems. Kongsberg [4], Sonardyne [5] and iXblue [6] are the market leaders.

Alternative methods proposed in the literature, e.g., in [2] are Simultaneous Localization And Mapping (SLAM) with Extended Kalman Filter (EKF) [7] [8], collaborative positioning [9], and methods including camera or light sensors data for localization [10].

\section{B. Underwater acoustic communication system}

In this paper, we propose to jointly design the underwater acoustic communication system and the localization system. Using UWA communications has advantages compared to other underwater communication technologies such as long range communication, no line of sight constraints, and costeffectiveness. On the other side, the UWA channel pose significant challenges well known by the UWA research community. For a moving AUV, a challenge is the estimation of the Doppler scaling factor that is related to the Doppler shift induced by the relative motion between transmitter and receiver. Doppler spreading is also sometimes experienced in UWA communication systems and this is due to time-varying scattering of the acoustic waves reaching the moving surface and/or spatially-varying sea-bottom conditions. The Doppler scaling factor, which is proportional to the relative velocity between transmitter and receiver, has to be estimated and compensated by the decoder (receiving side) in order to retrieve the information bits. In this paper, we then propose to feed the Doppler scaling factor (the relative velocity) estimation into the localization system and measure its experimental performance.

\section{Motivation}

This paper extends the work of [11] by introducing both range and velocity estimation into the localization method and we further run controlled environment experiments, e.g., in the water pool, to study its performance. Therefore, the proposed localization system integrates a a Range-Only Single Beacon (ROSB) localization method and a Doppler velocity estimation given by the communication system. As mentioned in the localization subsection, multiple methods co-exist and according to the need, budget and specifications of the mission the sensors data may be jointly exploited. Alternatively to the proposed system, the Doppler velocity could be estimated by a DVL. However, the proposed solution suggests to use the Doppler velocity estimated by the communication system, thus providing a more integrated solution for an AUV equipped with a UWA communication system than having to integrate an additional DVL.

The scenario consists of an AUV following a lemniscate trajectory around a geo-referenced buoy equipped with a subsea acoustic transponder sending periodically UWA data to the AUV. The experiment is performed in a 3 meter long and 1 meter large pool. Two mechanical arms, equipped with two transceivers, represent the buoy and the AUV. The movement induced Doppler shift, measured at the receiver (AUV side) is numerically inserted in each acoustic transmission performed in the water pool. The main goal is to accurately estimate the position of the AUV relative to the (known) the position of the transmitter.

The remainder of this article is organized such as follows: Section II describes the communication system model made of the transmitted signal and the receiver algorithms. Section III presents the dynamic model used in the experiments and experimental results are discussed in Section IV. Finally we conclude the paper in Section V.

\section{CommunicAtion System MODEL}

\section{A. Transmitted signal}

Data is transmitted by using the same signal as in [11]. Each frame is formed by 150 pilot symbols used for UWA channel estimation and 2518 Binary Phase Shift Keying (BPSK) data symbols carrying the information. They are pulse-shaped with a square-root raised cosine (SRRC) filter and then transposed around $f_{0}=23 \mathrm{kHz}$ in the band between $17 \mathrm{kHz}$ and $30 \mathrm{kHz}$. An out-of-band pure tone signal around $f_{p t}=11 \mathrm{kHz}$ is added to the useful signal in order to estimate very accurately motion induced Doppler shift [12]. The Power Spectral Density (PSD) of the transmitted signal is showed in Fig. 1.

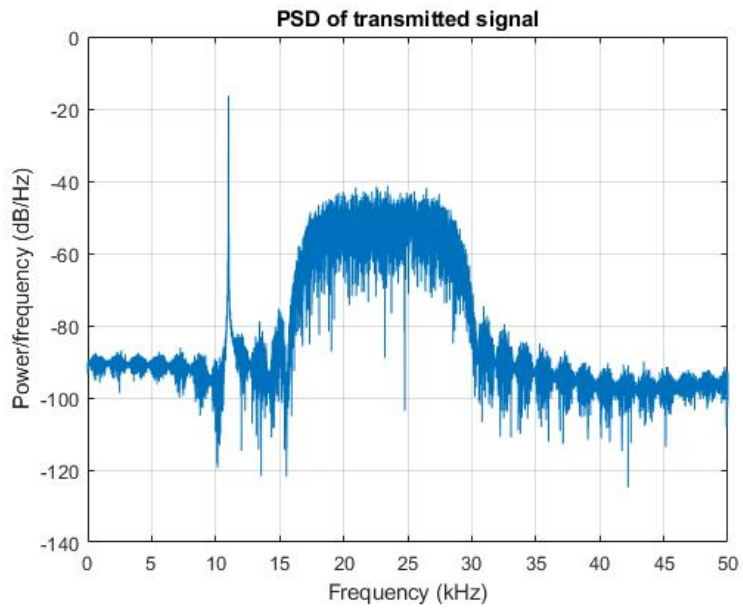

Fig. 1: PSD of typical transmitted UWA communication signal.

Since the receiver arm speed cannot be controlled, the overall continuum trajectory is sampled into 60 points. The arm representing the AUV moves through each point and at each point an UWA communication is performed. The range is then estimated by using this recorded signal, however the Doppler velocity is numerically emulated by resampling the pass-band recorded acoustic signal according 
to the speed induced Doppler scale factor, as described in [13].

\section{B. Receiver algorithm}

At the receiver, the demodulation process requires an estimation of the instantaneous Doppler shift. The chosen algorithm consists of extracting the Doppler shift from the phase derivative of the pure-tone baseband signal $\tilde{r}_{p t}(t)$ by computing the phase angle from two successive samples as follows:

$$
\hat{v}_{r}(k T)=\frac{c_{w}}{2 \pi T f_{p t}} \arg \left(\tilde{r}_{p t}(k T) \tilde{r}_{p t}^{*}((k-1) T)\right)
$$

where $c_{w}$ is the celerity of sound underwater, $T$ is the modulation symbol duration and $\hat{v}_{r}(k T)$ is the estimated Doppler velocity.

The range measurement is estimated at the receiver (AUV side) by the frame synchronization algorithm that realizes a cross-correlation between the received signal and the known transmitted pilot symbols. By assuming identical sampling frequency between buoy and the AUV, the distance is then evaluated according to the time of flight of the transmitted signal, as done in [3].

\section{DYNAMIC MODEL}

Let us assume that the AUV has a position $(x, y)$, a velocity $v$ and a heading angle $\theta_{h}$. This heading angle is considered to be known, e.g., estimated by a compass. The vehicle is controlled by acceleration and heading angle computed by a feedback linearization method. The reference anchor is assumed fixed with $\left(x_{\text {ref }}, y_{\text {ref }}\right)$ values. The most common trajectory for an AUV are survey patterns such as the "lawn mower" pattern or grid pattern, as described in [14] for a 3D space. We restrict our study to a 2D space, since the third dimension can be added by considering the measurements by pressure sensors. For this reason, we chose to focus on a Lemniscate with trajectory defined as:

$$
\left\{\begin{array}{l}
x_{d}=a \sin f_{1} t /\left(1+\cos f_{1} t^{2}\right) \\
y_{d}=a \sin f_{1} t \cos f_{1} t /\left(1+\cos f_{1} t^{2}\right)
\end{array}\right.
$$

The parameters of the equation of the trajectory are chosen in order to get a minimum error of the Doppler velocity estimation. As shown in [12] this means to maximize the Doppler shift, i.e., the speed of the AUV. We choose $a=0.6$ $\mathrm{m}$ and $f_{1}=1 \mathrm{~Hz}$ for $t$ ranging from 0 to 12 seconds with steps of $d t=0.2$ seconds, the speed is around $0.5 \mathrm{~m} / \mathrm{s}$.

\section{A. State space model}

The state vector is defined by $\mathbf{z}=\left(\begin{array}{lll}x & y & v\end{array}\right)^{T}$, the observation vector by $\mathbf{y}=\left(\begin{array}{ll}d_{\text {mes }} & v_{\text {mes }}\end{array}\right)^{T}$ with $d_{\text {mes }}$ the estimated range and $v_{\text {mes }}$ the estimated speed.

The state (3) and observation (4) equations at state $k$ are expressed as:

$$
\mathbf{z}_{\mathbf{k}}=f\left(\mathbf{z}_{\mathbf{k}-1}\right)+\mathbf{b}_{\mathbf{k}}
$$

$$
\mathbf{y}_{\mathbf{k}}=g\left(\mathbf{z}_{\mathbf{k}}\right)+\mathbf{d}_{\mathbf{k}}
$$

with $\mathbf{b}_{\mathbf{k}}$ representing the noise, $\mathbf{d}_{\mathbf{k}}$ is the observation error, $k$ represents the trajectory sample index, therefore it is a natural number from 1 to $k_{\max }=60$. Finally the state (5) and observation (6) functions are defined by :

$$
\begin{gathered}
\mathbf{f}\left(\mathbf{z}_{\mathbf{k}}\right)=\left(\begin{array}{lll}
x_{k}+v_{k} \cos \left(\theta_{h}\right) \cdot d t & y_{k}+v_{k} \sin \left(\theta_{h}\right) \cdot d t & v_{k}
\end{array}\right)^{T} \\
\mathbf{g}\left(\mathbf{z}_{\mathbf{k}}\right)=\left(\begin{array}{ll}
\sqrt{\left(x_{k}-x_{\mathrm{ref}}\right)^{2}+\left(y_{k}-y_{\mathrm{ref}}\right)^{2}} & v_{k}
\end{array}\right)^{T}
\end{gathered}
$$

Using speed only or range only estimation is also possible. The observation function vector has then 0 by nullifying either speed or range factor. Further in the paper we compare each solution in the pool.

\section{B. Kalman filter estimation}

Evaluating the position at stage $k$ given few measurements is the role of an estimator. The most commonly used estimator in localization methods is the EKF. For example in [15] the authors compare the performance of localization methods in a dynamic scenario based on EKF, Unscented Kalman Filter (UKF), Particle Filter (PF), and Maximum A Posteriori (MAP) with range only measurements and single beacon. In this paper, we consider an EKF algorithm which is described in [16]. The state transition matrix is

$$
\mathbf{A}=\left.\frac{\partial f\left(\mathbf{z}_{\mathbf{k}}\right)}{\partial z}\right|_{\mathbf{z}_{\mathbf{k}}}=\left(\begin{array}{ccc}
1 & 0 & d t \cdot \cos \left(\theta_{h}\right) \\
0 & 1 & d t \cdot \sin \left(\theta_{h}\right) \\
0 & 0 & 1
\end{array}\right)
$$

The Jacobian matrix A computed is independent from $k$. The observation transition matrix is

$$
\mathbf{H}=\left.\frac{\partial h\left(\mathbf{z}_{\mathbf{k}}\right)}{\partial z}\right|_{\mathbf{z}_{\mathbf{k}}}=\left(\begin{array}{c}
\frac{\left(x_{k}-x_{\mathrm{ref}}\right)}{\sqrt{\left(x_{k}-x_{\mathrm{ref}}\right)^{2}+\left(y_{k}-y_{\mathrm{ref}}\right)^{2}}} \\
\frac{\left(y_{k}-y_{\mathrm{ref}}\right)}{\sqrt{\left(x_{k}-x_{\mathrm{ref}}\right)^{2}+\left(y_{k}-y_{\mathrm{ref}}\right)^{2}}} \\
1
\end{array}\right)
$$

The command in acceleration and heading angle used in the feedback linearization method were not taken into account in the filter as opposed to what is done in [11], since we want to focus on speed and range measurements being fed to the estimator.

Furthermore, if the measurement is speed-only the two first lines of (8) are equal to 0 . In the opposite, if the measurement is range-only the last line of (8) is equal to 0 . The EKF also takes in input co-variance matrix $\mathbf{Q}$ and $\mathbf{R}$ for respectively $\mathbf{b}_{\mathrm{k}}$ and $\mathbf{d}_{\mathrm{k}}$.

$$
\begin{aligned}
& \mathbf{b}_{\mathbf{k}} \sim \mathcal{N}\left(\mathbf{0}_{3}, \mathbf{Q}=\sigma_{b}^{2} \mathbf{I}_{3}\right) \\
& \mathbf{d}_{\mathbf{k}} \sim \mathcal{N}\left(\mathbf{0}_{2}, \mathbf{R}=\sigma_{d}^{2} \mathbf{I}_{2}\right)
\end{aligned}
$$

where $\mathbf{0}=(0 \ldots 0)^{T}, \mathbf{I}_{d}$ is the identity matrix of dimension $d$. 


\section{Inducing the speed}

During the mission the AUV estimates its relative speed with motion induced Doppler shift. In practice, we are simulating this movement by inducing a Doppler shift depending on total velocity drift $v_{r}(t)=v_{r v}(t)+v_{r d}(t)+v_{r s}(t)$ where $v_{r d}(t)$ represents unintentional transmitter-receiver motion (like drifting), $v_{r v}(t)$ is the vehicular motion and $v_{r s}(t)$ describes the surface motion due to waves [17].

The receive passband signal can be modeled as:

$$
r(t)=\sum_{p=0}^{P-1} h_{p}(t) s\left(t-\tau_{p}(t)\right)+w(t)
$$

where $h_{p}(t)$ denotes the $p$-th coefficient of the UWA multipath channel impulse response varying in time, $s(t)$ is the transmitted passband signal, $w(t)$ the additive noise and $\tau_{p}(t)$ the time varying path delay modeled as:

$$
\tau_{p}(t)=\overline{\tau_{p}}-\int_{0}^{t} \frac{v_{r}(t)}{c_{w}} d t
$$

with $\overline{\tau_{p}}$ depicting the nominal path delay. Equation (13) can be rewritten as:

$$
r(t)=\sum_{p=0}^{P-1} h_{p}(t) \tilde{s}\left(t-\bar{\tau}_{p}\right)+w(t)
$$

with $\tilde{s}(t)$ motion induced communication signal obtained by resampling the pass-band transmitted $s(t)$ as follows:

$$
\tilde{s}(t)=s\left(t+\int_{0}^{t} \frac{v_{r}(t)}{c_{w}} d t\right)
$$

In practice, we compute the motion induced communication signal $\tilde{s}(t)$ by using (14) according to the desired speed $v_{r}(t)$ and use $\tilde{s}(t)$ as input of acoustic transmission scheme in water pool.

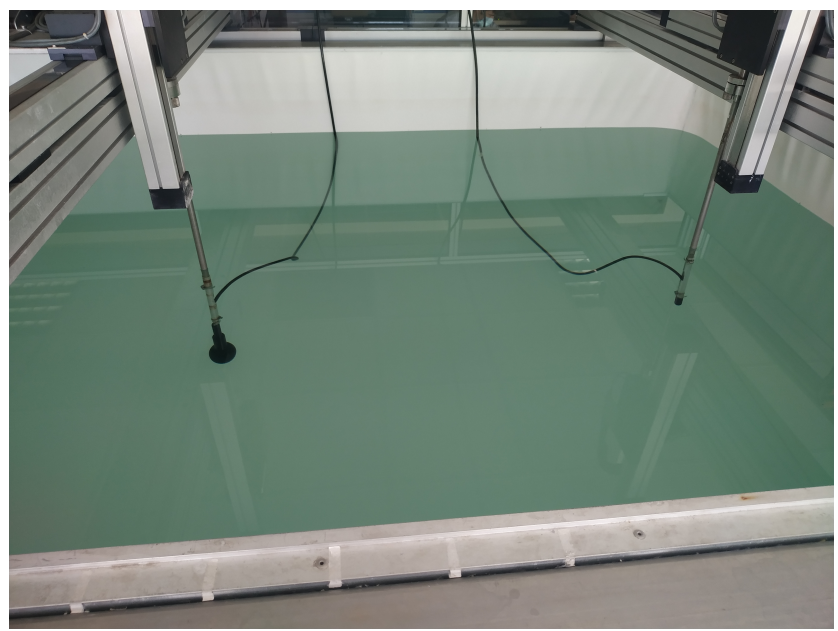

Fig. 2: Experiment pool with Neptune D/26 transmitter (left) and B\&K 8104 receiver (right).

\section{EXPERIMENTAL RESULTS}

The experimental set up is represented in Fig. 2. The arms are controlled in $(x, y)$ directions (also in depth that we fixed at $0,5 \mathrm{~m}$ for each arms and rotation which is not used here).

\section{A. Experiment parameters}

The range measurements used in range-only estimation and speed and range estimation are the same. The pool environment is characterized by several acoustic wall reflections, however lower noise levels than in subsea conditions. Celerity of sound $c_{w}$ is measured to $1480 \mathrm{~m} / \mathrm{s}$ in 0,5 meters depth. Figure 3 shows the measurement error ratio with respect to the actual speed and range (emulated and controlled through the mechanical arm, respectively). The distance computation from the correlation is slightly imprecise due to several acoustic waves reflections.

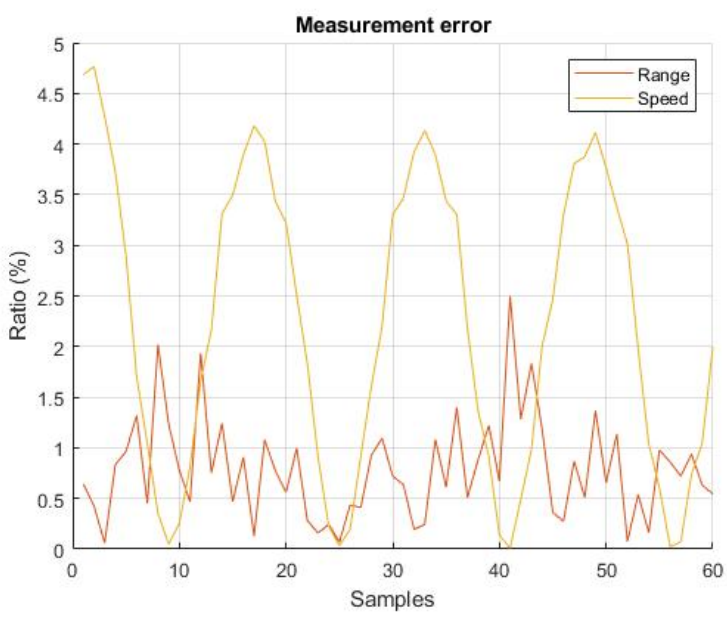

Fig. 3: Error ratio of the measurement with the true value

We initialize the Kalman filter estimator, $\hat{\mathbf{z}_{0}}$, at the actual initial coordinate $[-0.7 ; 0]$ since the AUV can access to its GPS position before beginning to submerge and start the mission underwater.

The covariance error matrices $\mathbf{Q}$ and $\mathbf{R}$ and $\mathbf{P}_{\mathbf{0}}$ are:

$$
\mathbf{Q}=10 \mathbf{I}_{3}, \mathbf{R}=15 \mathbf{I}_{2}, \mathbf{P}_{\mathbf{0}}=\mathbf{I}_{3} .
$$

$\mathbf{P}_{\mathbf{0}}$ is the initial state covariance matrix of the EKF.

In order to limit the phenomenon of acoustic reflection of the pool, a guard band of 5000 symbols is added to each 60 communications at the beginning of the signal. The reference anchor is at $\left(x_{\text {ref }}=0.7, y_{\text {ref }}=0\right)$.

\section{B. Results}

Figures 4 and 5 show the obtained experimental results in terms of estimated trajectory and positioning error statistics. As we can see, at the very beginning the speed-only estimation is the best but during the mission a consequent bias appears and the positioning estimation of the filter start to drift. This is an exaggerated fact that also held in classical INS DVL systems. Since the speed measurements are allegedly worse 
than those obtained by a DVL, the drifting bias is also worse. We observe the same phenomenon of offset growing with the time.

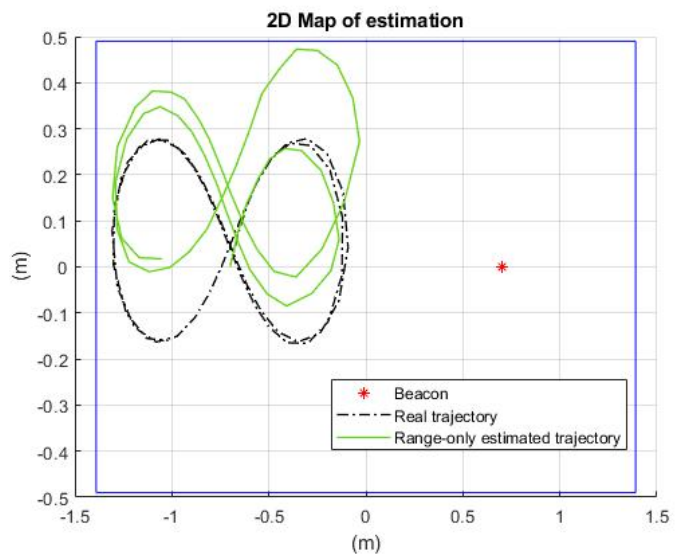

(a) Range-only measurements.

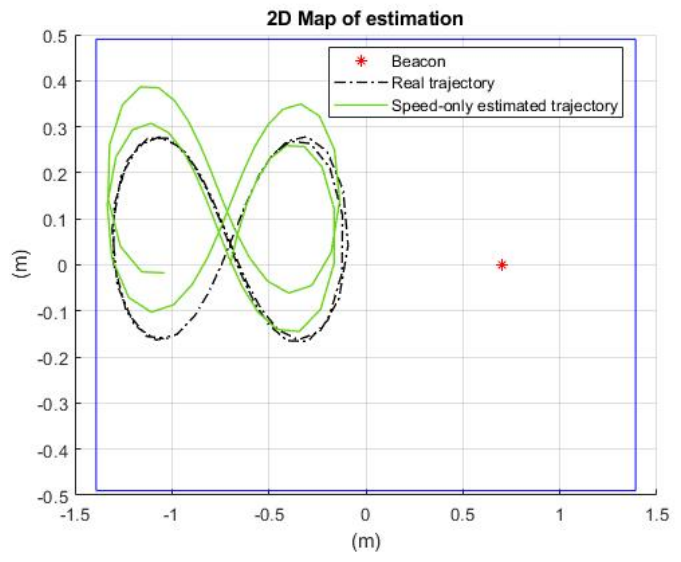

(b) Speed-only measurements.

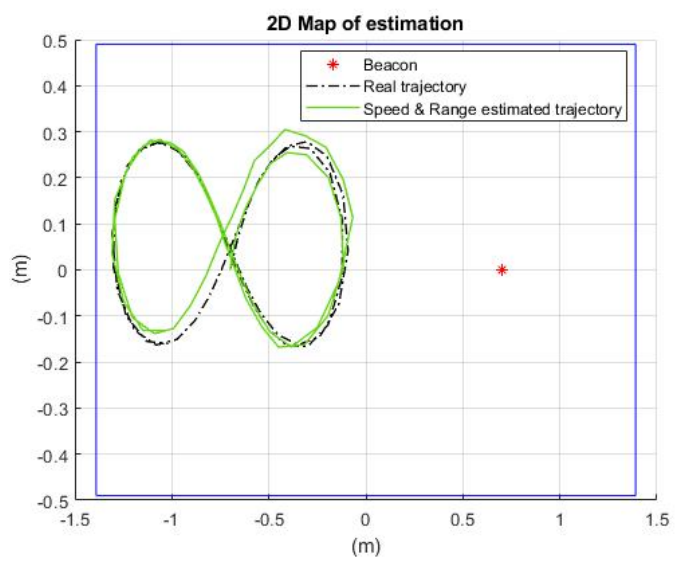

(c) Speed and Range measurements.

Fig. 4: Trajectory estimation, in green, using an Extended Kalman Filter and the same speed and range measured in (c) as in (a) and (b).
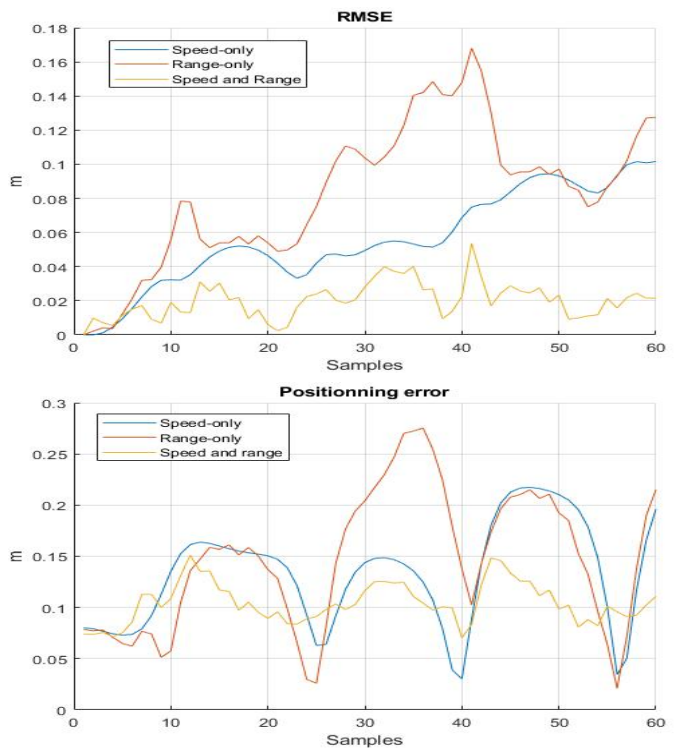

Fig. 5: Representation of error dispersion and positioning error.

The range-only estimation is neither precise (variance of the error) nor accurate (average error) because the Kalman Filter does not have the acceleration information and the range measurement are not accurate with respect to the size of the left side pool $\left(1,5 \mathrm{~m}^{2}\right)$. The measurement error is usually in the order of a centimetre, but it is significant enough. The proposed localization method that uses speed and range estimations, outperforms the speed only and range only localization methods in terms of accuracy (average positioning error). In particular, the proposed scheme has a constrained positioning error, unlike the speed-only localization method.

\section{CONCLUSION}

The use of cost-effective Doppler shift estimation computed after a received UWA communication in localization was presented in this paper. The Doppler shift measurement allow us to compute the speed of the AUV. Combined with classical beacon range localization, we perform an experiment of the proposed localization method. In a fairly small and reflective environment we showed that even with consequent offsets in measurements with real speed or range values, using Doppler shift estimation improves the localization estimation of the AUV by increasing overall accuracy and removing the speedonly estimation growing bias. Future work will focus on nonlinear estimators (e.g. PF, UKF) and grid pattern trajectory in a bigger underwater environment such as lake or sea.

\section{REFERENCES}

[1] M. Stojanovic and P.-P. J. Beaujean, "Acoustic Communication," in Springer Handbook of Ocean Engineering, M. R. Dhanak and N. I. Xiros, Eds. Springer International Publishing, 2016, pp. 359-386.

[2] J. González-García, A. Gómez-Espinosa, E. Cuan-Urquizo, L. G. García-Valdovinos, T. Salgado-Jiménez, and J. A. E. Cabello, "Autonomous underwater vehicles: Localization, navigation, and communication for collaborative missions," Applied Sciences, vol. 10, no. 4, 2020. [Online]. Available: https://www.mdpi.com/2076$3417 / 10 / 4 / 1256$ 
[3] H.-P. Tan, R. Diamant, W. K. G. Seah, and M. Waldmeyer, "A survey of techniques and challenges in underwater localization," vol. 38, no. 14, pp. 1663-1676, 2021. [Online]. Available: https://www.sciencedirect.com/science/article/pii/S0029801811001624

[4] Kongsberg website. [Online]. Available: https://www.kongsberg.com/

[5] Sonardyne website. [Online]. Available: https://www.sonardyne.com/

[6] Ixblue website. [Online]. Available: https://www.ixblue.com/

[7] D. Lee, D. Kim, S. Lee, H. Myung, and H.-T. Choi, "Experiments on localization of an auv using graph-based slam," in 2013 10th International Conference on Ubiquitous Robots and Ambient Intelligence (URAI), 2013, pp. 526-527.

[8] P. Newman and J. Leonard, "Pure range-only sub-sea slam," in 2003 IEEE International Conference on Robotics and Automation (Cat. No.03CH37422), vol. 2, 2003, pp. 1921-1926 vol.2.

[9] A. Caiti, V. Calabrò, T. Fabbri, D. Fenucci, and A. Munafò, "Underwater communication and distributed localization of auv teams," in 2013 MTS/IEEE OCEANS - Bergen, 2013, pp. 1-8.

[10] F. Eren, S. Pe'eri, M.-W. Thein, Y. Rzhanov, B. Celikkol, and M. R. Swift, "Position, orientation and velocity detection of unmanned underwater vehicles (uuvs) using an optical detector array," Sensors, vol. 17, no. 8, 2017. [Online]. Available: https://www.mdpi.com/1424$8220 / 17 / 8 / 1741$

[11] C. Aubry, P. Forjonel, P. Bouvet, A. Pottier, and Y. Auffret, "On the use of doppler-shift estimation for simultaneous underwater acoustic localization and communication," in OCEANS 2019 - Marseille, pp. 1-5.

[12] P. Kathiroli, P.-P. J. Beaujean, and N. Xiros, "Source speed estimation using a pilot tone in a high frequency acoustic modem," in OCEANS'11 MTS/IEEE KONA, 2011, pp. 1-8.

[13] P. A. van Walree, F.-X. Socheleau, R. Otnes, and T. Jenserud, "The watermark benchmark for underwater acoustic modulation schemes," IEEE Journal of Oceanic Engineering, vol. 42, no. 4, pp. 1007-1018, 2017.

[14] J. Das, F. Py, T. Maughan, T. O'Reilly, M. Messié, G. Sukhatme, and K. Rajan, "Coordinated sampling of dynamic oceanographic features with auvs and drifters," International Journal of Robotics Research, vol. 31, pp. 626-646, 042012.

[15] I. Masmitjà Rusiñol, "Acoustic underwater target tracking methods using autonomous vehicles," accepted: 2020-03-23T01:01:02Z Publisher: Universitat Politècnica de Catalunya. [Online]. Available: https://upcommons.upc.edu/handle/2117/180789

[16] Y. Kim and H. Bang, Introduction to Kalman Filter and Its Applications, 112018.

[17] P. Qarabaqi and M. Stojanovic, "Statistical characterization and computationally efficient modeling of a class of underwater acoustic communication channels," IEEE Journal of Oceanic Engineering, vol. 38 , no. 4, pp. 701-717, 2013. 